\title{
Changes of Internal State Are Expressed in Coherent Shifts of Neuromuscular Activity in Aplysia Feeding Behavior
}

\author{
Yuriy Zhurov, Alex Proekt, Klaudiusz R. Weiss, and Vladimir Brezina \\ Department of Physiology and Biophysics and Fishberg Research Center for Neurobiology, Mount Sinai School of Medicine, New York, New York 10029
}

The multitasking central pattern generator (CPG) that drives consummatory feeding behaviors of Aplysia can produce ingestive, egestive, and intermediate motor programs. External stimuli trigger the programs but, remarkably, do not directly specify which type of program is produced. Rather, recent work has proposed, the type of program is determined by the internal network state of the CPG that has developed in response to the previous history of the stimulation. Here we have tested a key prediction of this network-state hypothesis. If the network state has a real existence and governs real functional behavior, changes in the state should be seen as coherent, coordinated changes along many dimensions of interneuron and motor neuron activity, muscle contraction, and ultimately movement, that underlie functional behavior. In reduced neuromuscular preparations, we elicited repetitive motor programs by continued stimulation of the esophageal nerve while recording the firing of motor neurons $\mathrm{B} 8, \mathrm{~B} 15, \mathrm{~B} 16, \mathrm{~B} 4 / 5$, and $\mathrm{B} 48$, and contractions of the accessory radula closer and I7-I10 muscles that respectively close and open the animal's food-grasping organ, the radula. Using sonomicrometric techniques, we similarly recorded the movement of the radula in the complete buccal mass. Successive esophageal nerve programs indeed exhibited clear progressive changes in motor neuron firing, muscle contractions, and the phasing of radula movements within each cycle, from an initially intermediate or even ingestive character to a strongly egestive character. We conclude that the Aplysia feeding CPG really has a coherent internal network state whose dynamics are likely to be reflected in the real behavior of the animal.

Key words: behavioral plasticity; internal state; neuromuscular system; central pattern generator; motor programs; feeding behavior; Aplysia

\section{Introduction}

Like other cyclical, rhythmic behaviors, the consummatory feeding behaviors of Aplysia are driven by a central pattern generator (CPG). This multitasking CPG produces several types of motor programs that underlie ingestive feeding behaviors (biting, swallowing) as well as egestive behavior (rejection of swallowed inedible material) (Kupfermann, 1974; for review, see Elliott and Susswein, 2002). The CPG does not cycle spontaneously: each cycle is triggered by sensory stimuli. Because these stimuli are signals that food is present, or on the other hand that inedible material has been swallowed, it might be thought that they would also specify which type of motor program, ingestive or egestive, is produced in response. Remarkably, however, it appears that they do not do so directly. Proekt et al. (2004) have recently proposed that the state of the CPG network mediates between the stimulus and the response. Sensory stimuli act through the dynamics of the CPG to influence the evolution of the network state, but it is the state of the network that exists at any moment that determines the type of motor program that is produced (Proekt and Weiss, 2003; Proekt

\footnotetext{
Received Aug. 16, 2004; revised Dec. 9, 2004; accepted Dec. 19, 2004.

This work was funded by National Institutes of Health grants. We thank Dr. Jian Jing for advice on the sonomicrometric experiments and Dr. Sven Vilim for help with video digitization.

Correspondence should be addressed to Dr. Vladimir Brezina, Department of Physiology and Biophysics, Box 1218, Mount Sinai School of Medicine, 1 Gustave L. Levy Place, New York, NY 10029. E-mail: Vladimir.Brezina@mssm.edu.

DOI:10.1523/JNEUROSCI.3361-04.2005

Copyright $\odot 2005$ Society for Neuroscience $\quad$ 0270-6474/05/251268-13\$15.00/0
}

et al., 2004). With its slow dynamics and consequent history dependence, the network state has the character of a simple predictive, expectational, or intentional internal state (Proekt et al., 2004).

Successful execution of the feeding behaviors requires the coordinated contractions of the many muscles of the animal's feeding organ, the buccal mass (for review, see Hooper et al., 1999) (for relevant details, see Results). In each type of feeding behavior the muscles contract in different coordination, with different amplitudes and phase relationships. Each type of underlying motor program is consequently characterized by a different coordinated pattern of firing of motor neurons and CPG interneurons. If the network state governs the production of functional motor programs and behavior as proposed by Proekt et al. (2004), changes of the network state should therefore be seen not just in selected neurons, but as coherent, coordinated changes along many dimensions of interneuron, motor neuron, and muscle activity. However, Proekt et al. (2004) proposed the existence of the network state on the basis of evidence obtained primarily with just one motor neuron that is commonly used as a readout of the type of motor program, neuron B8. The network-state hypothesis predicts that, simultaneously with the changes of firing of B8 observed by Proekt et al. (2004), there should be changes in the firing of other motor neurons, and furthermore in the contractions of the muscles themselves, all coordinated to produce coherent changes in the type of feeding behavior.

The accessory radula closer (ARC, or I5) muscle (Cohen et al., 
1978) is the best studied buccal mass muscle. Much is known about its contractile properties, its motor neurons, and its participation in the different types of feeding behavior (for review, see Hooper et al., 1999; Brezina et al., 2003) (see Results). Furthermore, there is good knowledge also of its antagonist, the radula opener muscle complex I7-I10 (Evans et al., 1996), which the network-state hypothesis predicts might change in opposite ways to the ARC. Using this experimentally advantageous pair of antagonistic neuromuscular circuits as well as techniques to monitor the movements of the complete buccal mass, here we test, and widely confirm, the predictions of the network-state hypothesis.

\section{Materials and Methods}

This work used three principal neuromuscular preparations: a reduced buccal-ARC preparation, a reduced buccal-opener preparation, and the complete buccal mass with both ARC and opener components. A schematic diagram of the buccal ganglia and buccal mass is shown in Figure 1. Components of the reduced buccal-ARC preparation are marked in red, components of the reduced buccal-opener preparation in blue, and common components in green.

Esophageal motor programs in the reduced buccal-ARC preparation. The ARC experiments presented here were among those previously analyzed, for a different purpose, by Horn et al. (2004).

The preparation was the standard preparation used for recording motor neuron-elicited contractions of the ARC muscle (Cohen et al., 1978; Weiss et al., 1979; Horn et al., 2004), except combined with extracellular nerve recording and stimulation. Briefly, the preparation consisted of the bilateral buccal ganglia, the ARC muscles, and the connecting buccal nerves 3, through which the buccal motor neurons B15 and B16 innervate the ARC muscle. The cerebral ganglion, connected to the buccal ganglia by the cerebral-buccal connectives, was also retained. The buccal ganglia (but not the cerebral ganglion) were desheathed. The ARC muscles were split, each remaining connected to the rest of the preparation only through its buccal nerve 3 . Experiments were generally done on only one side of each preparation, although, if this failed, the other side was available. The muscle on the chosen side (the "ipsilateral" side) was pinned out in a separate subchamber and connected to an isotonic transducer (model 60-3000; Harvard Apparatus, Holliston, MA) to measure the length of the muscle with a light counterbalancing load. The ipsilateral motor neurons B15 and B16 were impaled with standard intracellular microelectrodes, and their membrane voltage was monitored with an intracellular amplifier (Axoclamp 2A/B; Axon Instruments, Union City, CA). Electrical activity in three buccal nerves, namely the I2 nerve, the ipsilateral buccal nerve 2, and the radula nerve (for nomenclature, see Cohen et al., 1978; Scott et al., 1991; Hurwitz et al., 1994), was recorded differentially through suction electrodes connected to an extracellular amplifier (Differential AC Amplifier model 1700; A-M Systems, Carlsborg, WA). The same amplifier, under the control of a separate stimulator (Grass S48/S88; Astro-Med, West Warwick, RI), was used to stimulate the ipsilateral esophageal nerve. All signals were sampled and recorded simultaneously by a computer using Digidata 1322A dataacquisition hardware and pClamp 8/9 software (Axon Instruments). Most experiments were done at $15-16^{\circ} \mathrm{C}$, although some were done at room temperature with no obvious difference in the phenomena of interest here.

The esophageal nerve stimulation consisted of a long train of regular voltage pulses with parameters adjusted to elicit identifiable motor programs (see below) at moderately frequent intervals while the stimulation continued. The individual voltage pulses were typically $7-10 \mathrm{~V}$ in amplitude, $7-15 \mathrm{~ms}$ in duration, delivered at $2-3 \mathrm{~Hz}$. Three minute "blocks" of this stimulation, separated by $7 \mathrm{~min}$ rest periods, were repeated as long as the motor programs continued to be elicited.

Esophageal motor programs in the reduced buccal-opener preparation. The opener preparation and experiments were identical to the ARC preparation and experiments just described, with the following exceptions. Instead of the ARC muscle, the opener muscle complex I7-I10 (Evans et al., 1996) was used. The I9 and I10 muscles on one side (the ipsilateral side), plus the single I8 muscle and both I7 muscles, were left interconnected, together with a small piece of the odontophore cartilage into which the I7 muscles insert. The isotonic transducer was attached to this piece of cartilage to record the contractions of the whole complex. The contralateral I9 and I10 muscles were cut, as were all nerves except the ipsilateral buccal nerve 3, which alone remained connecting the opener muscle complex with the buccal ganglia. Instead of motor neurons B15 and B16, intracellular recordings were made from the ipsilateral opener motor neurons B48 and B4/5 (i.e., either one of B4 or B5, which act indistinguishably on the opener muscle) (Evans et al., 1996). These neurons, too, innervate the muscle through buccal nerve 3 (Warman and Chiel, 1995; Evans et al., 1996). Extracellular recordings were made from the I2 and radula nerves and, for convenience, from the contralateral buccal nerve 2. Control recordings simultaneously from both buccal nerves 2 showed that motor program activity appeared similarly in the contralateral as in the ipsilateral nerve. The ipsilateral esophageal nerve was stimulated.

A pilot hybrid preparation. A few experiments (see Fig. 8) were performed in a reduced preparation in which an ARC muscle and the opener muscle complex were both retained, although split from each other and connected to the rest of the preparation by opposing buccal nerves 3 (the ARC side was considered to be the ipsilateral side), and attached to two separate isotonic transducers so that the contractions of both muscles could be recorded simultaneously. One ARC motor neuron on the ipsilateral side and one opener motor neuron on the contralateral side (i.e., the side on which the opener muscle remained connected) were recorded from intracellularly, the I2 nerve and the ipsilateral buccal nerve 2 extracellularly. Either the ipsilateral or the contralateral esophageal nerve was stimulated, or sometimes both, drawn into separate suction electrodes but stimulated identically in parallel. Control recordings simultaneously from the same neuron or nerve on the ipsilateral and contralateral sides showed that these three stimulation variants gave essentially identical results (i.e., stimulation of either esophageal nerve was sufficient to elicit normal, complete programs of similar strength simultaneously in both buccal ganglia), except that stimulation of both nerves sometimes appeared to elicit faster programs, with a shorter cycle period, than stimulation of just one nerve.

Analysis of data from the reduced preparations. Motor programs were identified by the presence of a characteristic coordinated pattern of electrical activity in the I2 nerve and buccal nerve 2, namely first a burst of at least moderately intense activity in the I 2 nerve, then, beginning soon after the end of the I 2 nerve burst, a characteristically shaped burst of intense activity in buccal nerve 2 (see Figs. 2, 5) (for previous use of these criteria, see Morton and Chiel, 1993a; Hurwitz et al., 1996; Nargeot et al., 1997; Kabotyanski et al., 1998; Jing and Weiss 2001, 2002; Morgan et al., 2002). The protraction phase of the program was defined to be coincident with the I2 nerve burst; the retraction phase was defined as lasting from the end of the I2 nerve burst to the end of the buccal nerve 2 burst. Precisely, a "motor program" was defined as each unit of these two phases, and a "cycle" as the motor program together with its preceding interprogram interval (see Fig. 2, bottom). Although other program features, for example, characteristic bursts of motor neuron B15, B16, B48, or B4/5 firing (see Figs. 2, 5), could be seen in the other recording channels, the definition in terms of the I2 nerve and buccal nerve 2 bursts was primary. When either burst was very weak or absent, no program was identified. When a program could not be thus identified, yet the other recording channels showed a strong program, the entire block of stimulation was rejected. Furthermore, only those blocks were accepted that had four or more identified motor programs, and at least some muscle contraction. Altogether, 458 programs in 60 blocks from 10 buccal-ARC preparations, and 752 programs in 94 blocks from 12 buccal-opener preparations, were accepted for further analysis.

Initial processing of the raw records was done in Clampfit (Axon Instruments). The programs were identified, and the beginning and end times of their protraction and retraction phases were marked by eye. This appeared to be sufficiently reliable as the changes in nerve activity were usually quite abrupt (see Fig. 2). Motor neuron B15, B16, B48, and B4/5 spike times were automatically tabulated using the threshold event detection module of Clampfit 9. Times of the largest class of units in the radula nerve (see Figs. 2, 5) were similarly tabulated to obtain motor neuron B8 spike times (Morton and Chiel, 1993b). 
Subsequent processing of the data, its collation across multiple blocks and preparations, and all statistical analysis was done in Mathematica (Wolfram Research, Champaign, IL). The motor neuron B15, B16, B48, and $\mathrm{B} 4 / 5$ spike times were converted to instantaneous firing frequency functions, assigning to each time point in an interspike interval the reciprocal of the duration of that interspike interval. The B8 spike times were processed similarly; because the radula nerve contains axons of all four B8 neurons (Morton and Chiel, 1993b), the B8 firing frequencies in this paper are not those of a single B8 neuron, but higher, aggregate frequencies. The instantaneous ARC or opener muscle contraction amplitude during any program was defined as instantaneous muscle length - muscle length at the beginning of the program; thus, contraction of the muscle is positive in sign. Because different preparations, with different muscle sizes and somewhat different loads, could differ significantly in their contraction amplitudes, all contraction amplitudes were normalized by the maximal amplitude reached in that particular preparation. Further details of the analysis are given in Results and in the figure legends.

Esophageal nerve-elicited radula movements in the complete buccal mass. The buccal mass preparation was similar to those previously used by, for example, Evans and Cropper (1998) and Orekhova et al. (2001). The entire buccal mass was removed from the animal, together with the buccal ganglia and the cerebral ganglion, and anchored dorsal-side up in a Sylgard-lined dish by pinning the lip tissue. The esophageal nerves were cut for stimulation, but other buccal nerves (not only the buccal nerves 3 but also buccal nerves 2 , the radula nerve, and the I2 nerve, all of which were cut in the reduced preparations) remained intact (the cerebralbuccal connectives were also intact.) Extracellular wire electrodes, glued into place, were used to record the electrical activity in one or both of the buccal nerves 2 . The ganglia were not desheathed. To gain access to the radula, an aperture was created by removing the esophagus where it emerges from the buccal mass. In some experiments the piezoelectric crystals (see below) were implanted through this aperture, and it was then sutured shut. In most experiments, however, the aperture was surgically enlarged to permit visualization of the entire radula surface (see Fig. 9, video frames). Comparisons in the same preparation showed that the movements of the radula were not obviously different in the two cases.

Movements of the radula were recorded using sonomicrometric techniques as described by Orekhova et al. (2001), with a commercially available (Sonometric Corporation, London, Ontario, Canada) digital ultrasonic system (TRX-4 with $15 \mu \mathrm{m}$ resolution enhancement) with three or four omnidirectional piezoelectric crystals $(0.7 \mathrm{~mm}$ diameter, attached to 42 gauge Teflon-coated copper wire) implanted in the preparation. To record radula closure and opening, two crystals were sutured laterally onto the surface of the left and right radula halves (see Fig. 9, video frames). To record protraction and retraction of the radula, a third crystal was positioned in the dish centrally just to the rear of the buccal mass, and often a fourth crystal centrally in front of the buccal mass. In addition, the movements of the radula were routinely videotaped.

Movements were elicited as in the reduced preparations, by electrically stimulating one or usually both of the esophageal nerves in 3 min blocks separated by $7 \mathrm{~min}$ rest periods. Additionally, in some experiments carbachol was applied to the cerebral ganglion, which in these experiments was enclosed in its own separate subchamber, to elicit ingestive movements as described by Susswein et al. (1996).

The data were analyzed along the same lines as in the reduced preparations. Motor programs were identified by the bursts of electrical activity in buccal nerve 2. Only those blocks of stimulation were accepted that had four or more identified programs and reasonably well defined protraction-retraction as well as open-close movements of the radula. Altogether 394 program cycles in 32 blocks from eight preparations were accepted for further analysis. Because different preparations, with different buccal mass sizes, differed greatly in the absolute amplitude of the radula movements, all movements processed for the average profiles in Figure 11 were first normalized onto a uniform scale from 0 to $100 \%$, where $0 \%$ was the minimum and $100 \%$ the maximum value of the protraction-retraction record, or of the open-close record, in that particular block of stimulation. To neutralize any artifactual drift in the records

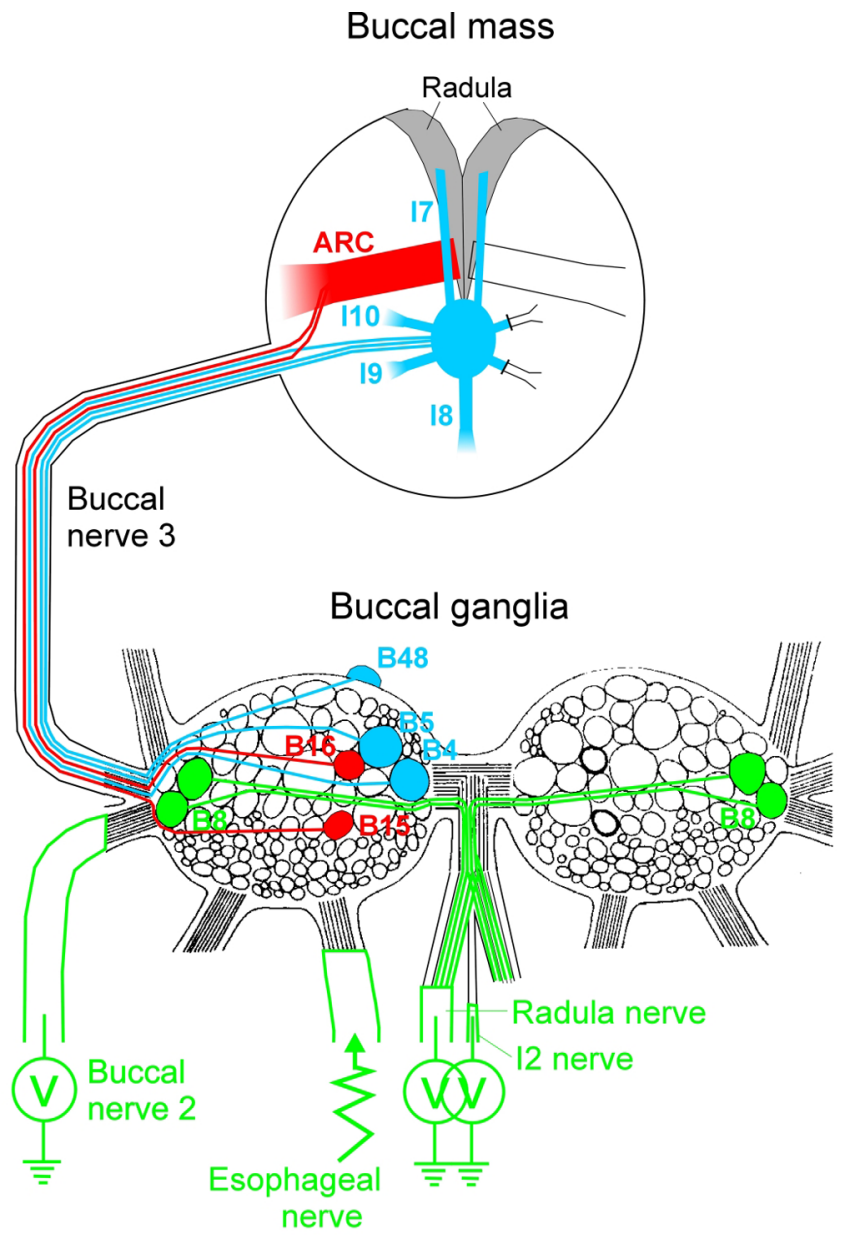

Figure 1. Schematic diagram of the buccal ganglia and buccal mass, indicating the components of the preparations used in this work. The diagram of the buccal ganglia is based on Cohen et al. (1978). The paths of innervation drawn within the buccal ganglia and the buccal mass are purely schematic.

resulting from imperfect removal of level shifts in the raw sonomicrometric signal (TRX-4 system manual; Sonometric Corporation), each individual cycle of movement was offset to the same level by subtracting the value of each record at the beginning of that particular cycle (at the beginning of the block of stimulation or, in subsequent cycles, at the beginning of the preceding interprogram interval). Later, after averaging, successive cycles were assembled by joining them end-to-end (see Fig. 11 ), in effect adding the (average) offsets back. Further details are given in Results and in the figure legends.

\section{Results}

We performed three main series of experiments, first studying the ARC neuromuscular circuit and the radula opener neuromuscular circuit separately in reduced preparations, then both together in the complete buccal mass.

\section{Esophageal motor programs in a reduced buccal-ARC neuromuscular preparation}

In the first series of experiments, we recorded the contractions of the ARC muscle and, with intracellular microelectrodes, the firing of its two motor neurons, B15 and B16 (Cohen et al., 1978), in the buccal ganglion (Fig. 1, red structures). We also recorded extracellularly the electrical activity in several buccal nerves (Fig. 1, green): the radula nerve, which carries axons of the four motor neurons B8 (Morton and Chiel, 1993b), and the I2 nerve and buccal nerve 2, whose patterns of activity are commonly used as 


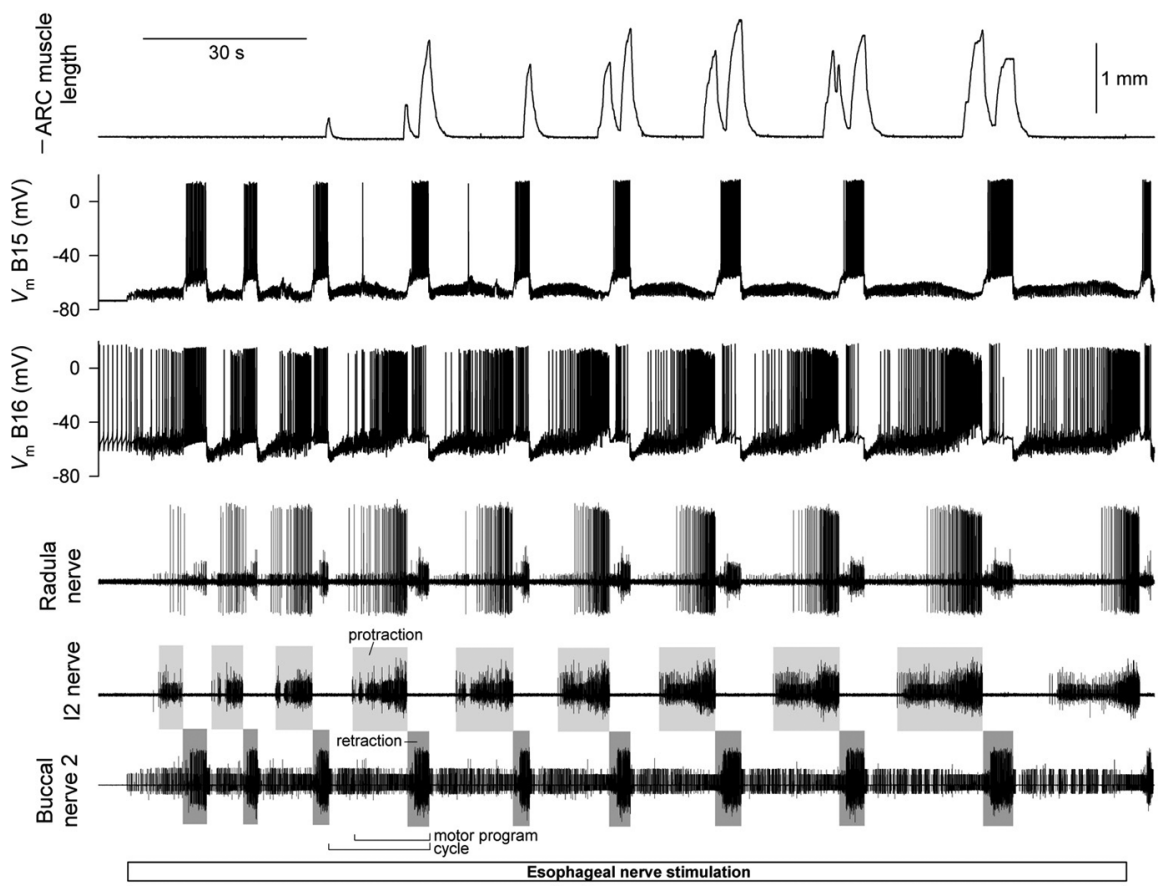

Figure 2. Typical 3 min block of motor programs elicited in a reduced buccal-ARC preparation by esophageal nerve stimulation. Simultaneous recording of (top to bottom) ARC muscle length, membrane voltage of the ARC motor neurons B15 and B16 (recorded intracellularly), and electrical activity in the radula nerve, 12 nerve, and buccal nerve 2 (recorded extracellularly) is shown. Note that the ARC muscle length is plotted so that decreasing length, increasing contraction, is upward. egestive behavior (Chiel et al., 1986; Cropper et al., 1990; Morton and Chiel, 1993a,b; Church and Lloyd, 1994). As expected therefore, direct electrical stimulation of the esophageal nerve can elicit egestive behavior or egestive motor programs, and has been used for this purpose in a number of experimental studies (Chiel et al., 1986; Susswein and Byrne, 1988; Morgan et al., 2002; Due et al., 2004; Horn et al., 2004). However, Proekt et al. (2004) recently observed that the motor programs elicited by esophageal nerve stimulation are not always egestive. Using an esophageal nerve stimulation protocol similar to our protocol here, they found that, although the stimulation was constant, successive motor programs exhibited a progressive change in character.

When recorded in the isolated nervous system, Aplysia feeding motor programs are usually classified as ingestive or egestive based on the firing pattern of the motor neurons B8. These motor neurons fire to close the radula (Morton and Chiel 1993b; Orekhova et al., 2001), which happens in retraction in ingestive behavior, but in protraction in egestive behavior (Kupfermann, 1974). Accordingly, if a markers respectively of the two major phases of each motor program that, in the intact animal, produce protraction and then retraction of the radula, the central food-grasping structure of the buccal mass (Kupfermann, 1974; Morton and Chiel, 1993a; Hurwitz et al., 1996; Nargeot et al., 1997; Kabotyanski et al., 1998; Jing and Weiss, 2001, 2002; Morgan et al., 2002).

To elicit motor programs, we electrically stimulated another of the buccal nerves, the esophageal nerve. In our standard protocol, we stimulated the nerve with brief shocks delivered at a moderate frequency $(\sim 3 \mathrm{~Hz})$ continuously for $3 \mathrm{~min}$, sufficiently long for the changes that we analyze in this paper to develop. All of the simultaneous channels recorded during one such "block" of stimulation are shown in Figure 2. As can be seen, multiple motor programs were typically elicited. The protraction phase of each program was determined from the activity in the I 2 nerve, the retraction phase from the activity in buccal nerve 2 (gray rectangles) (see Materials and Methods). In precise terms, as indicated in Figure 2, we defined a motor program as each unit of these two phases, and a "cycle" as the motor program together with its preceding interprogram interval. Each block was followed by a rest period of $7 \mathrm{~min}$, sufficiently long for recovery from the changes that developed during the block. During the rest period, with no external stimulation, the CPG was generally quiescent. Thereafter, another block was recorded. Altogether, we collected a dataset of 458 programs in 60 blocks ( $\sim 7.6$ programs per block on average) satisfying our criteria (see Materials and Methods) for further analysis.

Progressive evolution of the esophageal motor programs In the intact animal, the esophageal nerve is thought to convey satiety signals from the gut (Kuslansky et al., 1978) as well as sensory information signaling the presence of inedible food, a foreign object, or other mechanical irritation of the esophagus or buccal mass, all of which have been found experimentally to elicit motor program has a substantially higher frequency of firing of $\mathrm{B} 8$ in retraction than in protraction, it is taken to be an ingestive program; if the converse, an egestive program (Morton and Chiel, 1993a,b; Nargeot et al., 1997; Kabotyanski et al., 1998; Jing and Weiss, 2001, 2002; Morgan et al., 2002). Using this criterion, Proekt et al. (2004) observed that, after a period of inactivity of the CPG, the first esophageal motor programs were neither ingestive nor egestive, but intermediate, the apparent default state of the quiescent CPG. With continued stimulation, however, successive programs became progressively more egestive and eventually assumed a fully egestive character. Together with other evidence (see Discussion), this gave the basic idea of a historydependent internal state of the CPG that determines the character of the motor programs.

In our experiments here, we did not record from the motor neurons B8 intracellularly. However, their firing appears as the largest class of units in the extracellular record from the radula nerve (Morton and Chiel, 1993b). As Figure 2 shows (fourth record down), this large-unit activity showed, indeed, a characteristic progressive buildup in protraction, as in the work of Proekt et al. (2004). At the same time, progressive changes were evident also in other program dimensions (e.g., in the firing of motor neuron B16, and in the shape of the ARC muscle contractions, in Fig. 2).

A complicating factor was that, although the stimulation was constant, the motor programs were quite variable. Successive programs were often quite different from one another, to the extent that in some blocks any progressive trends were widely masked by the apparently random variability. The large variability is interesting in its own right and has already been fully analyzed in a previous report (Horn et al., 2004) (see Discussion) that isolated the variability by focusing on pairwise differences between adjacent programs. Here, on the other contrary, we wished 
to remove the variability to isolate the progressive trends. We did this by averaging.

Figure 3 shows the temporal profiles of the instantaneous firing frequencies of the motor neurons B8 (an aggregate of all four recorded in the radula nerve), $\mathrm{B} 15$, and B16, and the instantaneous ARC muscle contraction amplitude (columns from left to right), in the first, second, third... $>10$ th program of the 3 min block of esophageal nerve stimulation (rows from top to bottom) averaged from all 60 blocks in the dataset. At each (column, row) location, the first box represents protraction, the second box retraction. Examining the sequence of successive programs, the following principal features are evident: (1) Motor neuron B8 initially fired at low frequency in both protraction and retraction, then progressively increased its firing in protraction, but not in retraction. In the B8-based classification scheme for feeding motor programs, this represents a progressive shift from intermediate to egestive program character very like that found by Proekt et al. (2004). (2) Motor neuron B15 fired exclusively in retraction, increasing its firing somewhat in successive programs. (3) Motor neuron B16 initially fired more in retraction than in protraction, then progressively increased its firing in protraction while decreasing it in retraction. In B16, more obviously than in B8, there was therefore not merely an increase in firing, but an entire coordinated phase shift, from maximal firing in retraction to maximal firing in protraction. (4) The ARC muscle initially contracted little in either protraction or retraction, then built up its contractions, but differentially in the two phases. The contractions in retraction grew rapidly, over the first few programs, then slowly declined, whereas the contractions in protraction grew slowly and steadily. Consequently, as with the firing of B16, there was a phase shift of maximal contraction from retraction to protraction. (The resulting characteristic pattern of double peaks of contraction, with weight progressively shifting from the peak in retraction to the peak in protraction, is very obvious in the example in Fig. 2.) Indeed, the phase shift in ARC muscle contraction was presumably caused by the phase shift in B16 firing. The ARC muscle is contracted to any practical effect only by the two motor neurons B15 and B16 (Cohen et al., 1978; Jordan et al., 1993), furthermore only by those two that we were recording from in these experiments on the same side of the animal as the muscle (data not shown). Because B15 did not fire in protraction at all, the contractions in protraction, and their slow buildup, must have been produced entirely by the increasing firing of B16. The parallel slow decline of contractions in retraction in later programs, similarly, was presumably because of the decreasing firing of B16, as B15 firing was increasing. (Con-

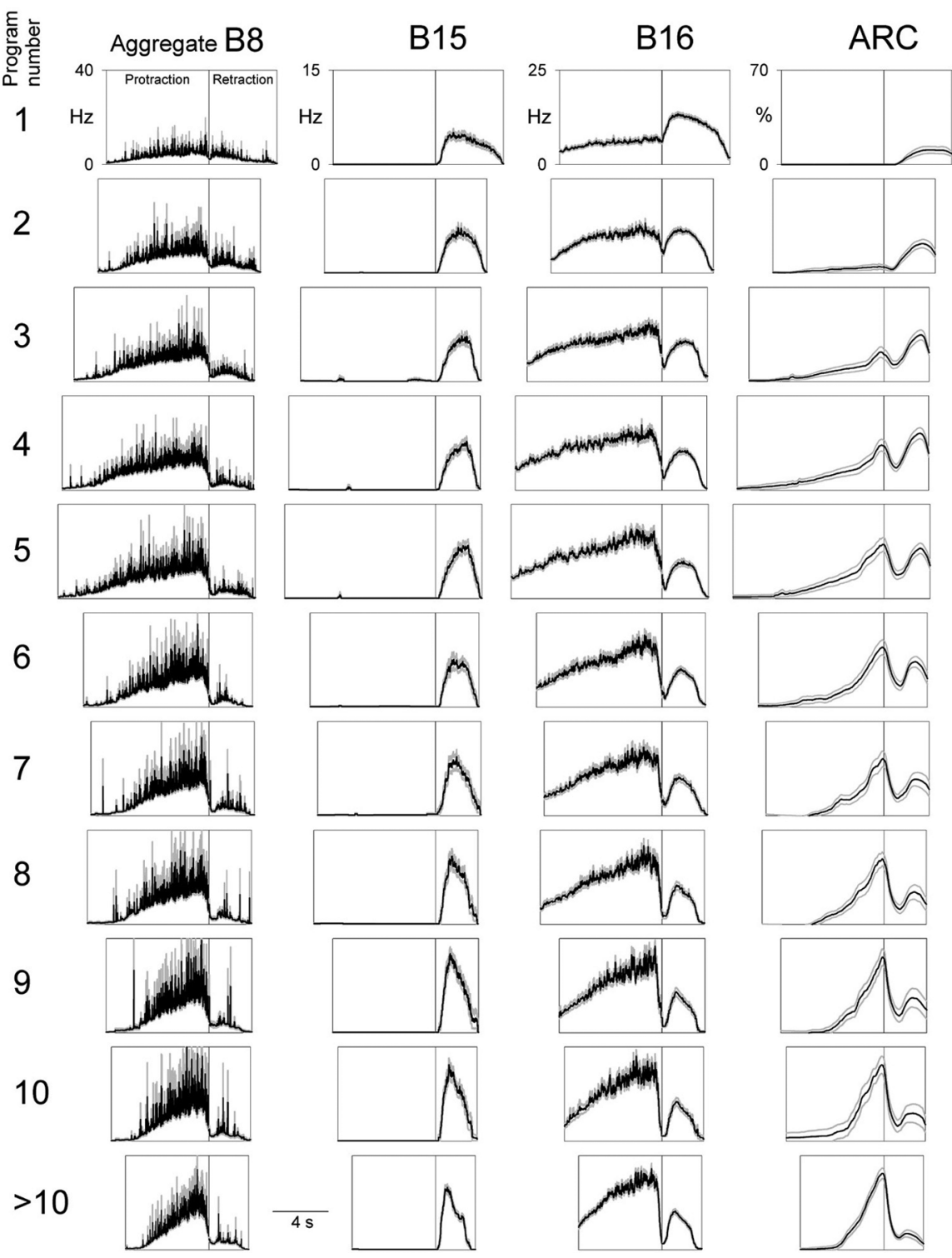

Figure 3. Evolution of successive esophageal motor programs in the reduced buccal-ARC preparation. Temporal profiles, averaged from the entire dataset of 458 programs recorded in 60 blocks in 10 preparations, of the instantaneous firing frequencies min block of esophageal nerve stimulation (rows from top to bottom) are shown. At each (column, row) location, the first box second box similarly represents retraction. Within each box, the black trace shows the mean instantaneous firing frequency or contraction amplitude, the gray envelope the SE. For successive rows, $n=60,60,60,60,46,39,29,25,18$, 17, and 44 (the last row pools programs 11-17 from blocks in which that many programs occurred).

versely, the rapid initial buildup of contractions in retraction was presumably caused by the increasing firing of B15, as B16 firing was decreasing.)

Despite averaging, the instantaneous firing frequency profile of B8 was conspicuously noisy (Fig. 3), reflecting the fact that, perhaps because of the many synaptic inputs converging on it, B8 fires with a very irregular spike pattern (Proekt et al., 2004). The B16 profile was similarly noisy, but only in protraction (Fig. 3): B16 switched from an irregular spike pattern in protraction to much more regular firing in retraction. B15, too, fired very regularly in retraction.

The simultaneous evolution of all of the measured program dimensions is summarized in Figure $4 A$, in a plot of the peak 
A ARC

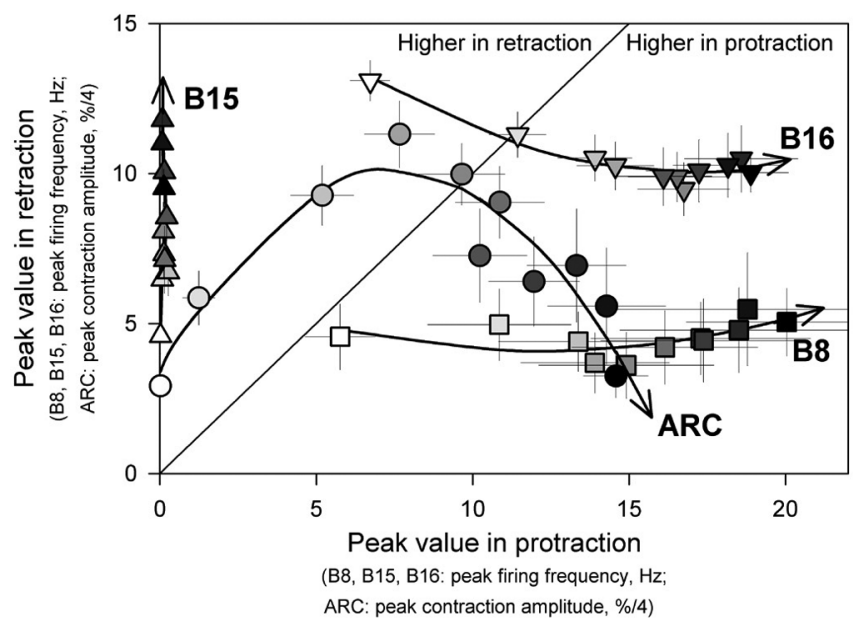

B Opener

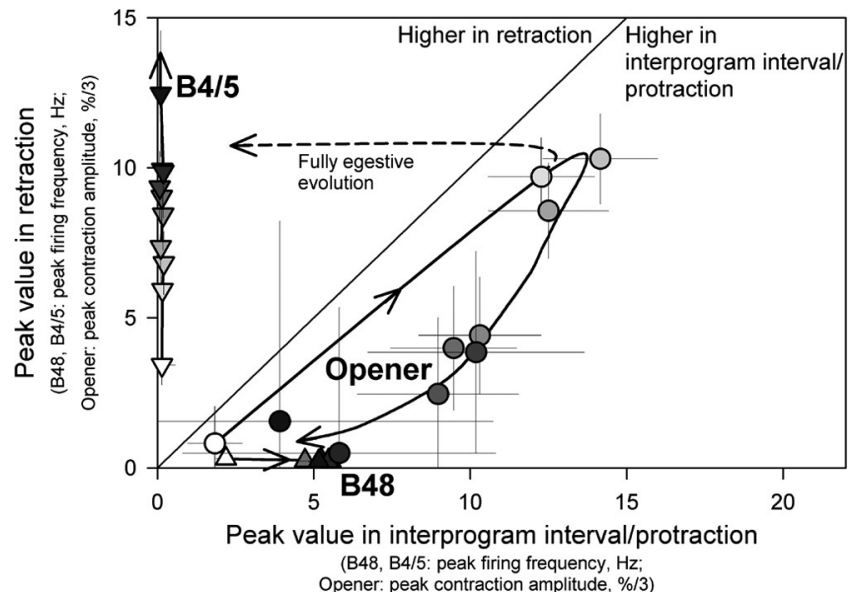

Figure 4. Summary of the multidimensional evolution of the esophageal motor programs. $A$, Dimensions measured in the reduced buccal-ARC preparation. Plotted are the peak firing frequencies and contraction amplitudes occurring in the protraction (abscissa) and retraction (ordinate) phases of the average profiles in Figure 3 . The profiles were first heavily smoothed so that only their broad envelopes were measured. Each point is shown as mean (i.e., the peak value of the smoothed mean profile in Fig. 3 ) \pm SE (the peak values of the smoothed SE profiles). Increasing fill density of the mean symbol indicates increasing program number. The curves were drawn essentially by eye. For the ARC values in retraction, the second distinct peak of contraction was measured. $\boldsymbol{B}$, Dimensions measured in the reduced buccal-opener preparation. Peak firing frequencies and contraction amplitudes occurring in the preceding interprogram interval or protraction phase (abscissa) and retraction phase (ordinate) of the average profiles in Figure 6. Otherwise as in $\boldsymbol{A}$. The B48 and B4/5 values were measured to reflect the main tendency of each phase: tails crossing from adjacent phases were ignored. For the curve labeled, "Fully egestive evolution," see Results, Predicted movements of the radula.

values of the profiles in Figure 3 (smoothed to remove the noise) in protraction versus retraction. Increasing fill density of the symbols, and the arrowed curves, indicate the successive programs. Crossing of the $45^{\circ}$ diagonal line marks a phase shift. Clear phase shifts from retraction to protraction can be seen in the firing of motor neuron B16 and contractions of the ARC muscle. Motor neuron B8 undergoes a similar evolution, although not formally a phase shift.

Because the ARC muscle participates in closing the radula (Cohen et al., 1978; Orekhova et al., 2001), the phase shifts in its contractions and in the underlying B16 firing represent, like the evolution of B8 firing, a shift from an intermediate or even somewhat ingestive to an egestive character of the motor programs, and furthermore of the muscle contractions that the programs control.

\section{Esophageal motor programs in a reduced buccal-} opener preparation

In the second series of experiments, we elicited esophageal motor programs in an identical manner but recorded the contractions of the radula opener muscle complex I7-I10 and the firing of its motor neurons, B48 and B4/5 (Evans et al., 1996) (Fig. 1, blue structures). Figure 5 shows a typical 3 min block of these motor programs. Altogether we collected a dataset of 752 programs in 94 blocks ( $\sim 8.0$ programs per block on average). Figure 6 shows the temporal profiles of the instantaneous firing frequencies of B48 and $\mathrm{B} 4 / 5$ and the instantaneous opener muscle contraction amplitude (columns from left to right) in the first, second, third. . . 10th program of the block (rows from top to bottom) averaged from this entire dataset. Because motor neuron B48 (unlike B4/5, or B15 or B16) fired most during the interval between programs (Fig. 5), Figure 6 shows at each (column, row) location three boxes, representing respectively the preceding interprogram interval (this box is therefore missing from the first row), protraction, and retraction. Examining the sequence of successive programs, the following principal features are evident: (1) Motor neuron B48 invariably exhibited a characteristic profound hyperpolarization in retraction, during which it fired no spikes at all (Fig. 5). At the end of retraction the firing appeared to rebound to reach its highest frequency, which was then maintained in a gently decreasing plateau through the interprogram interval and the protraction of the next program. In the very first protraction, perhaps because there was no previous hyperpolarization to rebound from, the firing frequency was low, but after the characteristic profile was established at the first retraction, it remained essentially constant in all successive programs. (2) Motor neuron B4/5 fired exclusively in retraction, increasing its firing steadily in successive programs (as is very obvious in the example in Fig. 5). (3) The opener muscle contracted little in the first program; the contractions then built up rapidly over the next one or two programs before slowly declining in later programs. (The slow decline was the clear average trend, but the contractions also became more erratic and irregular: the gray curves in Fig. 6 show the envelope of the SE.) Throughout, the contraction occurred mostly during the interprogram interval and protraction. Although the contraction often spilled over well into retraction, there appeared to be little active contraction in retraction (but see below). Presumably most of the contraction was produced by the firing of B48. Indeed, the raw records often exhibited a peak of contraction corresponding to the rebound of B48 firing at the end of retraction (Fig. 5).

As the activity of B48 and the opener muscle in the interprogram interval makes clear, although, based on precedent and convenience, we have here defined the motor program to consist of just the protraction and retraction phases visible in the $\mathrm{I} 2$ nerve and buccal nerve 2 recordings, organized activity in fact extends significantly beyond these phases, well into the "interprogram interval" (Weiss et al., 1986; Jing et al., 2004).

The simultaneous evolution of all of the program dimensions measured in the buccal-opener preparation is summarized in Figure $4 B$, in the same way as the dimensions measured in the ARC preparation were summarized in Figure $4 A$. 
Predicted movements of the radula The ARC and opener muscles are antagonists: the ARC muscle participates in closure of the radula, and the opener muscle participates in its opening (Cohen et al., 1978; Evans et al., 1996; Orekhova et al., 2001). Both muscles participate also in other movements performed by the buccal mass, but here too they are antagonists (Orekhova et al., 2001). To predict, at least to a first approximation, the open-close movement of the radula, as in previous work (Weiss et al., 1992; Brezina and Weiss, 2000), we simply summed the oppositely directed contraction waveforms of the two muscles.

Figure 7 shows the average ARC and opener contraction profiles from Figures 3 and 6 (top traces), and then the predicted movement of the radula (bottom trace), in program 2, early in the sequence of programs (left), and program 9, late in the sequence (right). In program 2, the radula is predicted to be open in protraction, then to close in retraction. This is therefore at least an intermediate or even an ingestive pattern of radula movement: the radula protracts open, closes around food, then retracts closed to draw the food into the esophagus (Kupfermann, 1974; Morton and Chiel, 1993a). In program 9, in contrast, the contraction of the ARC muscle, and therefore the closure of the radula, has shifted to protraction. This is a distinct shift toward the egestive pattern of radula movement, wherein the radula protracts closed around inedible material to push it out of the mouth. For a fully egestive pattern, however, the radula should then open in retraction to leave the expelled material outside and not draw it back in (Morton and Chiel, 1993a). This opening does not occur in Figure 7 because, in most of our experiments, the opener muscle failed to contract significantly in retraction. The hypothetical fully egestive pattern of opener contraction and radula movement in a late program such as program 9 is sketched by the dashed curves labeled "Fully egestive contraction/movement" in Figure 7, right. The hypothetical full evolution of opener contractions from intermediate or ingestive to egestive is sketched by the curve labeled "Fully egestive evolution" in Figure $4 B$. The full evolution would require a complete phase shift of opener contractions from protraction to retraction, complementing the observed shift of ARC contractions from retraction to protraction.

Evans et al. (1996) suggested that the opener muscle may be driven to contract to some extent during retraction by the firing of the motor neurons B4/5. Indeed, we found here that the motor neurons $\mathrm{B} 4 / 5$ fire at precisely the right time in retraction, and with precisely the progressively increasing firing frequency in successive programs, to drive the hypothesized opener contractions (compare the B4/5 profiles in Fig. 6 with the dashed curves in Fig. 7). Yet, in most experiments, we did not see such contractions. There are several possible explanations. The single central opener muscle complex is innervated by the motor neurons B4/5 in both buccal ganglia, through both buccal nerves 3 . But in our reduced buccal-opener preparations one of the nerves was cut, depriving the opener muscle of half of its innervation (Fig. 1). Furthermore, Evans et al. (1996) found that the opener muscle contracts much more vigorously when it is first stretched and showed that it is in fact stretched by the contractions of its antagonist, the ARC muscle. Large stretches of the opener are also predicted by biomechanical models of the buccal mass (Drushel et al., 2002; Neustadter et al., 2002). The potentiating effect of stretch persists for several seconds after the stretch (Evans et al., 1996). Thus in the intact, mechanically coupled system, the large contractions of the ARC muscle (and other coactivated muscles) at the end of protraction in later programs may greatly potentiate a rebound contraction of the opener muscle during retraction. But in our reduced buccal-opener preparations, this mechanical coupling was gone.

In fact, there were sometimes contractions of the opener muscle in retraction, precisely as hypothesized, although in such a small proportion of preparations $(\sim 10 \%)$ that these contractions were not evident in the averaged profiles in Figure 6 . Figure 8 shows the results from one such preparation. This was one of a small number of pilot hybrid preparations in which we simultaneously recorded both ARC and opener contractions (see Materials and Methods). We do not believe, however, that the appearance of opener contractions was connected to the difference in preparation: this preparation was still reduced in both of the ways noted above, and similar contractions were seen in some openeronly preparations. Figure $8 \mathrm{~A}$ shows the first five motor programs from a representative block of esophageal nerve stimulation. Contractions of the ARC muscle exhibited their usual buildup and phase shift (third record down). Simultaneously, contractions of the opener muscle appeared in retraction and progressively built up in successive programs (fourth record down). On the other hand, the opener contracted little in protraction in this particular preparation. Although we did not record from the motor neurons B4/5 in this preparation, these opener contractions were clearly not driven by B48 firing (second record down) but rather reflected the usual firing profile of B4/5 (Fig. 6). Summing the ARC and opener contractions predicted alternating closures 


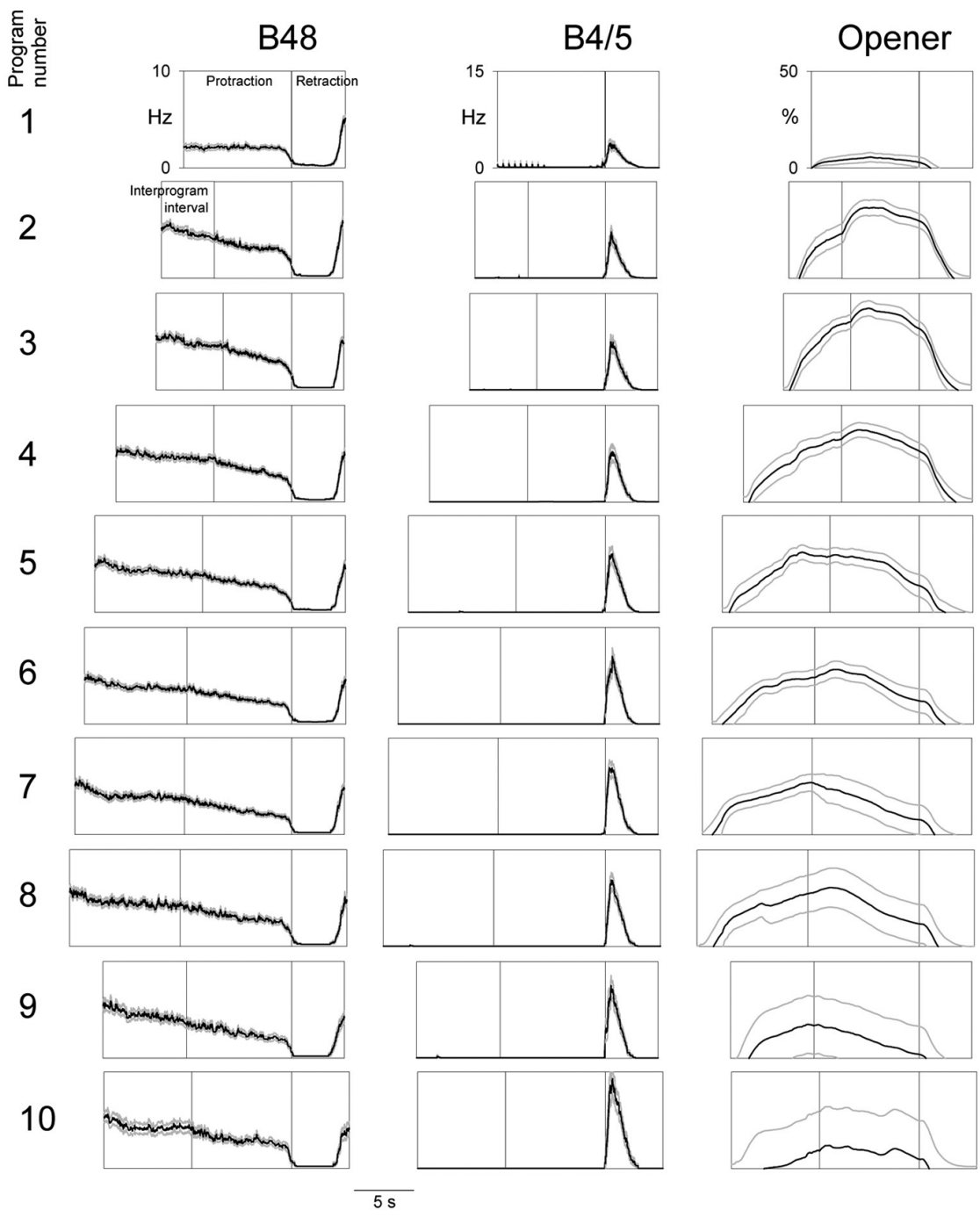

Figure 6. Evolution of successive esophageal motor programs in the reduced buccal-opener preparation. Similar to Figure 3. At each (column, row) location, the first box represents the preceding interprogram interval (this box is therefore missing from the first row), the second box protraction, and the third box retraction. Profiles were averaged from the entire dataset of 752 programs recorded in 94 blocks in 12 preparations. For successive rows, $n=94,94,94,94,90,86,73,59,40$, and 28.

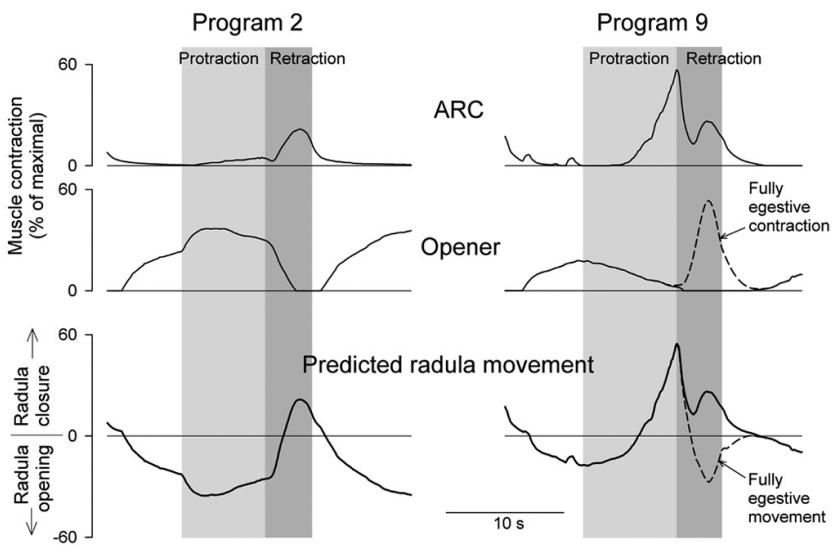

Figure 7. Evolution of predicted movements of the radula. The mean profiles of ARC and opener muscle contraction amplitude in programs 2 (left) and 9 (right) from Figures 3 and 6 (top 2 traces), and the predicted movement of the radula obtained by subtracting the opener trace from the ARC trace (bottom trace). For each program, the preceding interprogram interval, the protraction phase, the retraction phase, and the following interprogram interval are shown in order, with the duration of each phase set to the mean of the durations in Figure 3 and 6 . For the curves labeled, "Fully egestive contraction/movement," see Results, Predicted movements of the radula. and openings of the radula of progressively increasing amplitude in successive programs, shifting indeed from an intermediate or ingestive pattern in program 1 to a robust, fully egestive pattern by program 5 (Fig. $8 A$, bottom record). Figure $8 B$ shows profiles of the ARC and opener contractions and the predicted movement of the radula in program 9 , averaged from eight blocks recorded in this preparation. Note the marked resemblance of these profiles to the fully egestive profiles hypothesized in Figure 7, right.

\section{Radula movements in the complete buccal mass}

The preceding section predicted certain patterns of movement of the radula, but at the same time suggested that such predictions must be treated with caution because it is difficult to extrapolate from the contractions of the individual muscles in reduced preparations to the complete, mechanically complex buccal mass. Not only is it difficult to be sure how the individual contractions combine to give the overall movement, but the contractions in reduced preparations might in some respects differ from those in the complete buccal mass.

To test the predictions, therefore, in the third series of experiments we directly studied radula movements in the complete, largely intact buccal mass. We merely opened an aperture in the dorsal wall of the buccal mass to expose the radula, to be able to videotape it during the experiment and to implant on its lateral halves a pair of piezoelectric crystals for sonomicrometric recording (Fig. 9, first video frame). Sonomicrometry was previously used in the Aplysia buccal mass by Orekhova et al. (2001). In this technique, each crystal alternately emits and detects ultrasound pulses, switching at high frequency. Using the fixed speed of sound in an aqueous medium, the time elapsed between the emission of a pulse by one crystal, and its detection by another can be converted to the distance between the crystals, for a real-time mapping of the distances between all of the crystals in the system. The two crystals that we implanted across the radula halves recorded the closing and opening of the radula. Another crystal or pair of crystals, placed centrally in front of and to the rear of the buccal mass, recorded movement orthogonally with respect to the first pair of crystals, that is, protraction and retraction of the radula. Simultaneously, we monitored the underlying motor programs by recording the electrical activity in buccal nerve 2. In other respects the experiments were done as in the reduced preparations with blocks of esophageal nerve stimulation. Altogether, we collected a dataset of 394 programs in 32 blocks ( $\sim 12.3$ programs per block on average).

Figure 9 shows a typical block from the dataset. The dashed gray curve in the main plot shows the protraction-retraction movement of the radula (protraction up, retraction down); the solid black curve shows the open-close movement (closure up, 
opening down). The three video frames above show the radula in its neutral position, near the peak of protraction and closure, and near the peak of retraction and opening in one representative cycle. However, the movements can be much better appreciated in the complete video record, digitized at http://fulcrum.physbio.mssm.edu/ $\sim$ seaslug/RadulaMovement.html.

In agreement with the association of motor program phases with particular buccal nerve activities that Morton and Chiel (1993a) first described and many others (including ourselves in the reduced preparations) have since used, each significant retraction was associated with a burst of activity in buccal nerve 2 (Fig. 9, light gray record). However, the burst often began well before the movement: because of the relatively slow activation of the buccal mass muscles (Figs. 2, 5), peripheral "muscle time" was delayed with respect to central "neuronal time." In our analysis, we therefore used the bursts only as an initial guide to the presence of the successive motor programs and corresponding cycles of movement: the precise limits of each cycle were then derived from the movement records themselves. Specifically, we distinguished the segment during which the radula was generally protracting and that during which it was generally retracting in each cycle (for details, see Fig. 11 legend). The former corresponded to the protraction phase combined with the preceding interprogram interval, the latter to the retraction phase, of the motor programs in the reduced preparations. These segments are marked respectively by the filled and empty rectangles in the gray strip at the bottom of Figure 9. Similarly, we distinguished in the open-close movement record segments during which the radula was closing and those during which it was opening. These are marked respectively by the filled and empty rectangles in the black strip in Figure 9.

Ingestive and egestive movements should now be distinguishable by their opposite phasing of the protraction-retraction and open-close movements with respect to each other. In ingestive movements, the radula should be open during protraction and closed during retraction, in egestive movements the converse. Figure 10 illustrates fully developed examples of these two types of movement patterns. Fully egestive movements (Fig. 10A) were routinely recorded toward the end of most of the blocks of esophageal nerve stimulation. For comparison, fully ingestive patterns (Fig. $10 \mathrm{~B}$ ) were obtained by application of carbachol onto the cerebral ganglion, a treatment that has been well documented to elicit ingestive (specifically, biting) motor programs and behavior (Susswein et al., 1996).

Finally, Figure 11 presents the evolution of the successive cycles of radula movement during the block of esophageal nerve stimulation, in the form of summary profiles averaged from all 32 blocks in the dataset, as in Figures 3 and 6 except joined end-toend. In terms of the relative phasing of the protraction-retraction and open-close movements, we see in Figure 11 (as well as in the individual example in Fig. 9) that there was, indeed, a clear shift from an intermediate or even ingestive pattern over the first sev- eral cycles to a fully egestive pattern later in the block. Interestingly, in the fully developed egestive pattern, the closure of the radula was not exactly in phase with protraction but often exhibited a noticeable phase lead (Figs. 9, 11); similar phase leads or lags have been documented in the behavior of intact animals (Friedman et al., 2002) (S. C. Rosen, personal communication). Accompanying the ingestive to egestive shift, there was a progressive buildup of the amplitude of the movements. As we hypothesized from the results in the reduced preparations but could not conclusively show there, there was robust opening of the radula in each cycle of egestive movement. Last, the more ingestive cycles at the beginning of each block were superimposed on a general protraction of the radula (as were the ingestive cycles elicited by carbachol; data not shown), whereas the later egestive cycles were superimposed on a general retraction (Figs. 9, 11). This, too, was very reminiscent of observations made in more intact preparations and intact animals (Kupfermann, 1974; Weiss et al., 1986; Susswein et al., 1996; Evans and Cropper, 1998).

Altogether, therefore, it appeared that the radula movements in the complete buccal mass retained much of their character seen in intact animals, and did indeed undergo the coherent evolution from the intermediate or ingestive to the fully egestive pattern predicted with continued esophageal nerve stimulation by the network state hypothesis of Proekt et al. (2004).

\section{Discussion}

Our work here builds on that of Proekt et al. (2004), whose argument, therefore, is worth recapitulating somewhat more fully. Proekt et al. (2004) presented four lines of evidence. (1) With the esophageal nerve stimulation, Proekt et al. (2004) observed that the motor programs produced by the Aplysia feeding CPG evolved from an intermediate to a fully egestive character. (2) 


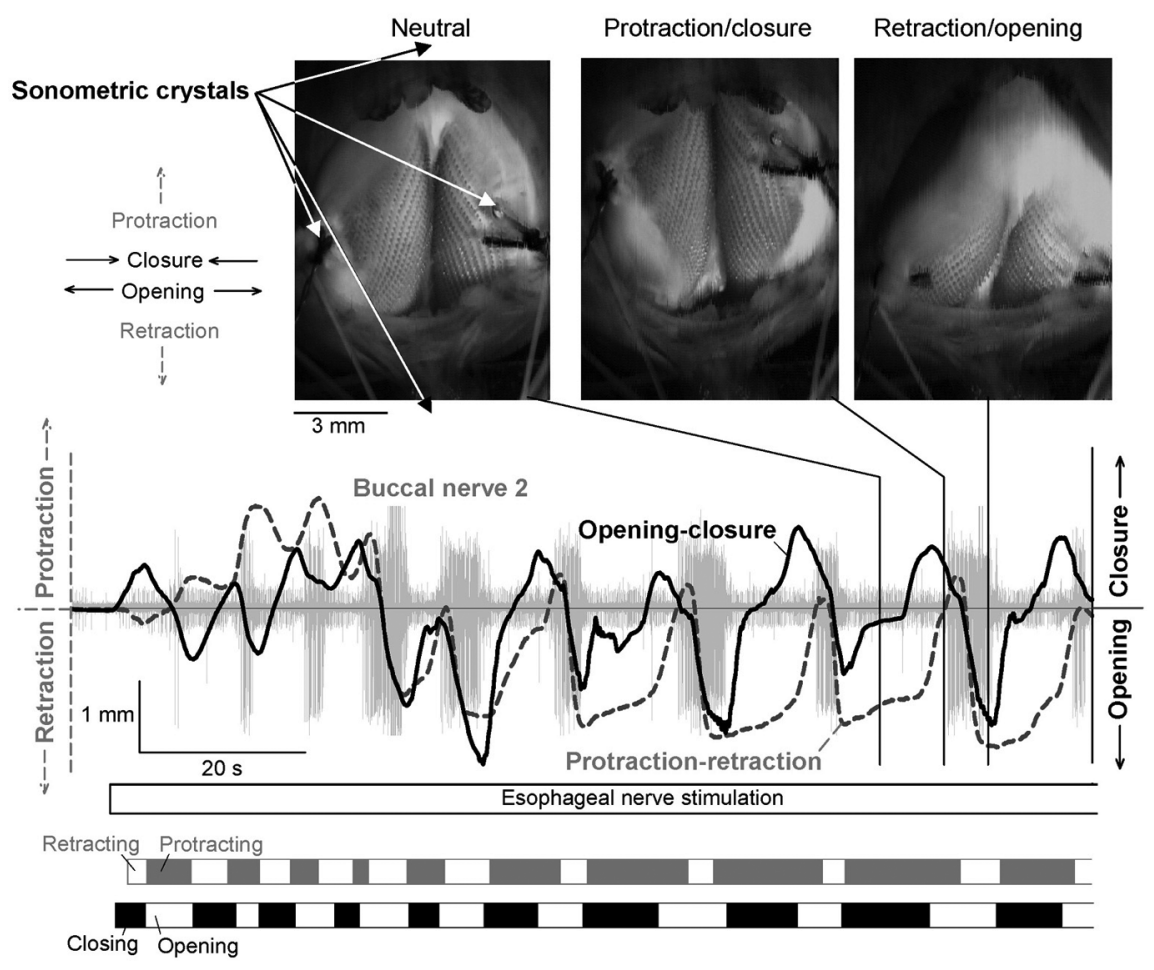

Figure 9. Typical movements of the radula elicited by esophageal nerve stimulation in the complete buccal mass. The three video frames are from a videotape of the experiment (digitized at http://fulcrum.physbio.mssm.edu/ seaslug/RadulaMovement.html), showing a dorsal view of the buccal mass with the toothed radula surface exposed. Four piezoelectric crystals for sonomicrometric recording of movement were placed as indicated by the arrows in the first frame. Two crystals, sutured laterally onto the radula halves to record closure and opening of the radula, can be seen; two other crystals, positioned centrally in front (above the video frame in the orientation shown) and to the rear (below the video frame) of the buccal mass to record protraction and retraction of the radula, were just out of the frame of the picture. The directions of movement in protraction, retraction, closure, and opening are indicated to the left of the first video frame. The graph shows a continuous readout of the distance between the two latera crystals (solid black curve) and between one of the lateral and one of the central crystals (dashed gray curve; the 4 lateral-central crystal pairings provided redundant information) during the first $\sim 2 \mathrm{~min}$ of a $3 \mathrm{~min}$ block of esophageal nerve stimulation. A simultaneous recording of electrical activity in buccal nerve 2 is also shown (light gray). The two strips at the bottom divide the block into segments with the same general direction of radula movement (see Results, Radula movements in the complete bucca mass). The three video frames, showing the radula in its neutral position, near the peak of protraction and closure, and near the peak of retraction and opening in one representative cycle, were obtained approximately at the times indicated in the graph by the three vertical black lines.

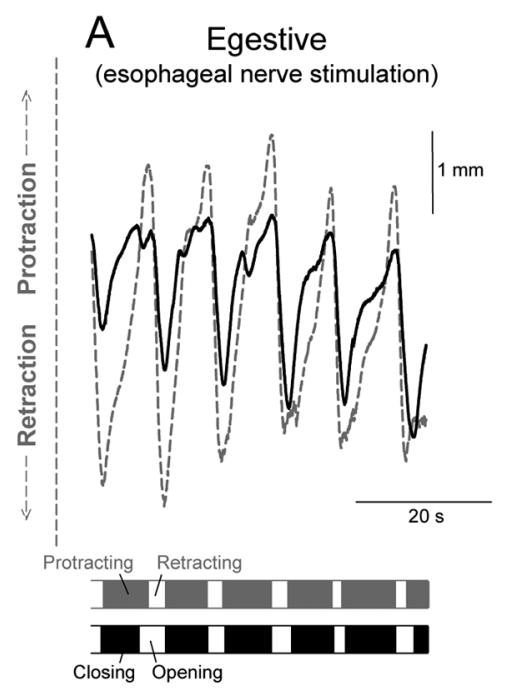

B
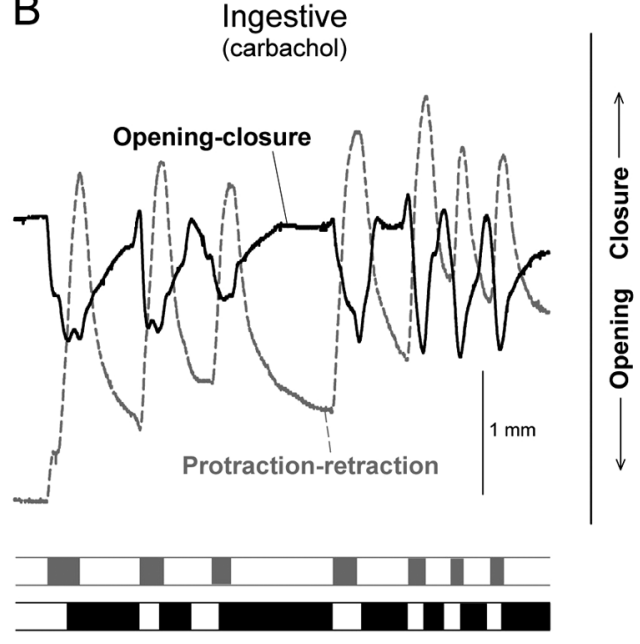

Figure 10. Contrast of fully egestive and fully ingestive patterns of radula movement. $A$, Egestive pattern, recorded toward the end of a 3 min block of esophageal nerve stimulation as in Figure 9 . $\boldsymbol{B}$, Ingestive pattern, elicited by application of $2 \mathrm{~mm}$ carbachol onto the cerebral ganglion. The records are offset arbitrarily relative to each other in this figure.
Similarly, with continued stimulation of interneuron CBI-2, a neuron that is believed to be part of a different, "ingestive" sensory input pathway, the programs evolved in the opposite, ingestive direction. (3) After the programs had been made fully egestive by esophageal nerve stimulation, the first programs subsequently elicited by CBI- 2 stimulation were not ingestive, but likewise fully egestive, indeed indistinguishable from those produced by the esophageal nerve stimulation. In other words, the preceding esophageal nerve stimulation switched the character of the CBI-2-elicited programs from ingestive to egestive, strongly suggesting that the changes in program character were not mediated by mechanisms localized in the input pathways themselves, but rather occurring downstream of their interaction, presumably in the CPG. (4) Finally, Proekt et al. (2004) demonstrated changes in efficacy of an identified synapse within the CPG that appear to mediate at least some of these changes in program character. Altogether, Proekt et al. (2004) argued that these observations reveal the existence of a history-dependent internal network state of the feeding CPG. Sensory stimuli, such as are presumably mimicked by the esophageal nerve and CBI-2 stimulation, trigger the CPG to produce motor programs and simultaneously act through the dynamics of the CPG to shape the progressive evolution of the network state, but it is the network state that exists at any moment that determines the character of the motor program, ingestive, egestive, or intermediate, that is produced.

In this paper we have aimed to test just one, but fundamental, prediction of this concept. Proekt et al. (2004) worked only in vitro, with the isolated ganglia that contain the feeding CPG, and furthermore with just one motor neuron, B8, whose firing pattern is conventionally used as an in vitro indicator of the degree of ingestiveness or egestiveness of motor programs. However, if the internal state of the CPG has a real existence and governs real functional behavior, as proposed by Proekt et al. (2004), changes in the state should be seen not just in one motor neuron, but as coherent, coordinated changes along the many dimensions of interneuron and motor neuron activity, muscle contraction, and ultimately movement, that underlie functional behavior. Here [working for simplicity with just the first paradigm of Proekt et al. (2004), the esophageal nerve stimulation] we have provided considerable evidence that such coherent, multidimensional change indeed occurs. Figure 4, for example, is a representation of it. 


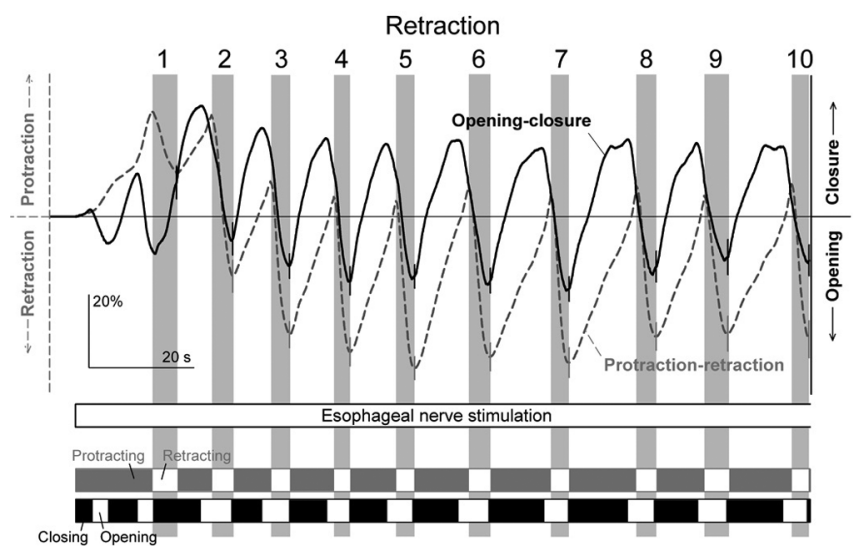

Figure 11. Evolution of successive cycles of radula movement elicited by esophageal nerve stimulation in the complete buccal mass. These are summary profiles averaged from the entire dataset of 394 cycles recorded in 32 blocks in eight preparations, as in Figures 3 and 6 except here joined end-to-end. Within each block, candidate cycles were first identified by the presence of a burst of electrical activity in buccal nerve 2, beginning just before the retraction movement in that cycle (Fig. 9) (see Results, Radula movements in the complete buccal mass). The bursts were used only to identify the presence of a cycle; the precise limits of the cycle were then derived from the movement records themselves. Specifically, the boundaries between successive cycles were defined to be the minima in the protraction-retraction record (i.e., peak retractions) between the beginnings of successive bursts of buccal nerve 2 activity; the first cycle was defined as beginning at the start of the esophageal nerve stimulation. Within each cycle, the retraction phase was defined as lasting from the maximum in the protraction-retraction record (i.e., peak protraction) to the final minimum; the rest of the cycle was therefore the protraction phase combined with the preceding interprogram interval. After normalization of the amplitude of the movement records in each block and cycle (amplitude is consequently expressed as percentage; see Materials and Methods), these two parts of each successive cycle were averaged separately over all the blocks in the dataset; the average profiles were scaled temporally to reflect the mean duration of each part; finally, the average profiles were joined end-to-end so that each successive part began at the amplitude of the end of the preceding part. The dashed gray and solid black curves are the mean values; the error bars show the SE, for clarity only at the boundaries between cycles. The vertical gray rectangles mark the retraction phases of successive cycles. For each cycle, $n=27-32$; only the first 10 cycles are shown.

It is worth stressing that the result could well have been different. First, stimulation of the esophageal nerve presumably mimics only part of the sensory stimulus set that normally reaches the CPG in the intact animal, and so could have produced only fragmentary, incoherent change, just the change in the firing of motor neuron B8 that was observed by Proekt et al. (2004), for instance, and nothing more. The fact that the partial stimulus is nevertheless able to elicit a complete, organized response supports the view of Proekt et al. (2004), which is indeed implicit in the concept of the CPG itself, that the state of the CPG is truly internal, organized by the circuitry within it. Second, because the "neuromuscular transform" from neural activity to muscle contractions and movement is complex, with possible degeneracy at several levels (Brezina et al., 2000; Brezina and Weiss, 2000; Drushel et al., 2002; Neustadter et al., 2002), the changes in neural activity could have complemented each other to leave muscle contractions or movement unchanged. Our results show, however, that there are changes in muscle contractions and movement.

At the neuronal level (Figs. 3, 4, 6), we have confirmed the result of Proekt et al. (2004) that, with continued esophageal nerve stimulation, motor neuron B8 firing frequency increases in protraction but not in retraction. In addition, we found that motor neuron B16 undergoes a similar but even more extreme change, increasing its firing in protraction while decreasing it in retraction for a complete phase shift in its firing. Motor neurons $\mathrm{B} 15$ and B4/5 increase their firing in retraction while not firing at all in protraction. Motor neuron B48, which fires in the interprogram interval and in protraction, undergoes little change. In this work we did not study the circuit mechanisms that might underlie these coordinated changes, but the known circuitry of the Aplysia feeding system (for review, see Cropper et al., 2004) includes a number of candidate mechanisms. For example, B4/5 inhibits both B8 and B16, and the increasing firing of B4/5 in the retraction phase of successive motor programs may well contribute to the decreasing firing of B8 and B16 as well as to the temporal profile of this firing with its distinctive notch at the protraction-retraction boundary (compare the B8, B16, and B4/5 columns of Figs. 3 and 6) (Kabotyanski et al., 1998; Jing and Weiss, 2001; Morgan et al., 2002).

The increasing firing of the radula closer motor neurons B8 and $\mathrm{B} 16$ in protraction and the increasing firing of the radula opener motor neurons B4/5 in retraction all suggest evolution of the motor programs in a more egestive direction. However, what determines the functional character of motor programs is the phasing of the muscle contractions that they produce, and in view of the complexity of the neuromuscular transform, this is best checked by examining the contractions themselves. Here we have indeed found a clear progressive evolution of the contractions of the ARC muscle, a radula closer, from retraction to protraction (Figs. 3, 4A), and, at least in some preparations, an evolution of the contractions of the radula opener muscle complex I7-I10 from protraction to retraction (Fig. 8), in both cases from an ingestive to an egestive pattern of muscle contraction.

However, most likely because of the unavoidable mechanical simplification of our reduced preparations, we could not demonstrate the phase changes in the radula opener contractions in all preparations. Furthermore, the biomechanics of the intact buccal mass are not yet well understood, and it is by no means clear precisely how the contractions of individual muscles such as the ARC and the radula opener complex contribute to the overall movement. Other muscles may also participate in radula closure and opening, and the functional significance of contractions may be context-dependent (Drushel et al., 2002; Neustadter et al., 2002). For these reasons it was necessary in this study to go up one more level of organization, to the actual movements of the radula in the complete buccal mass. We have confirmed that these movements are, indeed, initially small and have an ingestive phasing between radula closure and opening and its protraction and retraction in each cycle, but with continued esophageal nerve stimulation progressively evolve to large movements with egestive phasing (Figs. 9, 11). Because the complete buccal mass is already a nearly intact preparation, it seems very likely that a similar evolution will be found to occur in completely intact, freely feeding animals.

One caveat remains at this point: because esophageal nerve stimuli are presumably only part of the sensory stimulus set in the intact animal, we do not know that in the intact animal there will be precisely these changes in motor programs, muscle contractions, and movements. A large body of work has built up a strong, albeit circumstantial, case for thinking that esophageal nerveelicited motor programs are a reasonable in vitro counterpart of one type, hitherto thought to be always egestive, of in vivo feeding behavior (Susswein and Byrne, 1988; Morton and Chiel, 1993a,b; Church and Lloyd, 1994; Hurwitz et al., 1996; Nargeot et al., 1997; Kabotyanski et al., 2000; Brembs et al., 2004). Nevertheless, clearly the phenomena must now be reproduced in intact animals. 
Intact animals have, in fact, long been known to exhibit a phenomenon termed food-induced arousal, a progressive buildup of feeding movements in the ingestive direction under continued food stimulation (Kupfermann, 1974; Susswein et al., 1978; Elliott and Susswein, 2002). The work of Proekt et al. (2004) and our work here strongly suggest that all these phenomena are related instances of the same general class, driven by the dynamics of the network state of the feeding CPG.

Altogether, then, we can at least provisionally conclude that a coherent multidimensional state of the CPG exists and is very likely expressed in the actual feeding movements of the animal. What might be its behavioral significance? Proekt et al. (2004) have argued that, with its slow dynamics and consequent history dependence, the state of the CPG has the character of a simple predictive, expectational, or intentional internal state that extrapolates from past to future events. Because stimuli, such as esophageal nerve stimulation, are inherently ambiguous at any particular moment, they must be integrated over time to determine their true significance and to develop the correct response. Once developed, the response is likely to remain correct for some time because the component of the environment, for instance, food of a particular type, that gave rise to the stimulus and elicited the response tends to persist for some time. Around this integrated prediction, there is then the large random cycle-to-cycle variability that was studied by Horn et al. (2004), but averaged from our data here. This fast variability, also apparently generated by the CPG, has been proposed to implement a trial-anderror search mechanism through the parameter space of the feeding movements around the integrated prediction, a strategy that is optimal for the feeding animal in the face of environmental variability and uncertainty (Horn et al., 2004; Brezina et al., 2005). Approximately, we may think of the slow and fast dynamics as computing, respectively, the current best prediction of the environment and the range of uncertainty of that prediction. Both components of the dynamics of the internal network state of the CPG are thus matched to aspects of the environment (Horn et al., 2004; Proekt et al., 2004; Brezina et al., 2005). Now that our work here has shown that the internal state indeed governs coherent feeding movements, it should be possible to critically test these ideas with the real feeding behavior of intact Aplysia.

\section{References}

Brembs B, Baxter DA, Byrne JH (2004) Extending in vitro conditioning in Aplysia to analyze operant and classical conditioning in the same preparation. Learn Mem 11:412-420.

Brezina V, Weiss KR (2000) The neuromuscular transform constrains the production of functional rhythmic behaviors. J Neurophysiol 83:232-259.

Brezina V, Orekhova IV, Weiss KR (2000) The neuromuscular transform: the dynamic, nonlinear link between motor neuron firing patterns and muscle contraction in rhythmic behaviors. J Neurophysiol 83:207-231.

Brezina V, Orekhova IV, Weiss KR (2003) Neuromuscular modulation in Aplysia. I. Dynamic model. J Neurophysiol 90:2592-2612.

Brezina V, Horn CC, Weiss KR (2005) Modeling neuromuscular modulation in Aplysia. III. Interaction of central motor commands and peripheral modulatory state for optimal behavior. J Neurophysiol, in press.

Chiel HJ, Weiss KR, Kupfermann I (1986) An identified histaminergic neuron modulates feeding motor circuitry in Aplysia. J Neurosci 6:2427-2450.

Church PJ, Lloyd PE (1994) Activity of multiple identified motor neurons recorded intracellularly during evoked feedinglike motor programs in Aplysia. J Neurophysiol 72:1794-1809.

Cohen JL, Weiss KR, Kupfermann I (1978) Motor control of buccal muscles in Aplysia. J Neurophysiol 41:157-180.

Cropper EC, Kupfermann I, Weiss KR (1990) Differential firing patterns of the peptide-containing cholinergic motor neurons B15 and B16 during feeding behavior in Aplysia. Brain Res 522:176-179.

Cropper EC, Evans CG, Hurwitz I, Jing J, Proekt A, Romero A, Rosen SC (2004) Feeding neural networks in the mollusc Aplysia. Neurosignals 13:70-86.

Drushel RF, Sutton GP, Neustadter DM, Mangan EV, Adams BW, Crago PE, Chiel HJ (2002) Radula-centric and odontophore-centric kinematic models of swallowing in Aplysia californica. J Exp Biol 205:2029-2051.

Due MR, Jing J, Weiss KR (2004) Dopaminergic contributions to modulatory functions of a dual-transmitter interneuron in Aplysia. Neurosci Lett 358:53-57.

Elliott CJH, Susswein AJ (2002) Comparative neuroethology of feeding control in molluscs. J Exp Biol 205:877-896.

Evans CG, Cropper EC (1998) Proprioceptive input to feeding motor programs in Aplysia. J Neurosci 18:8016-8031.

Evans CG, Rosen S, Kupfermann I, Weiss KR, Cropper EC (1996) Characterization of a radula opener neuromuscular system in Aplysia. J Neurophysiol 76:1267-1281.

Friedman AK, Rosen SC, Kupfermann I (2002) Rasp vs. grasp: parametric differences between two modes of ingestive feeding behavior in Aplysia. Soc Neurosci Abstr 28:67.26.

Hooper SL, Brezina V, Cropper EC, Weiss KR (1999) Flexibility of muscle control by modulation of muscle properties. In: Beyond neurotransmission: neuromodulation and its importance for information processing (Katz PS, ed), pp 241-274. Oxford: Oxford UP.

Horn CC, Zhurov Y, Orekhova IV, Proekt A, Kupfermann I, Weiss KR, Brezina V (2004) Cycle-to-cycle variability of neuromuscular activity in Aplysia feeding behavior. J Neurophysiol 92:157-180.

Hurwitz I, Goldstein RS, Susswein AJ (1994) Compartmentalization of pattern-initiation and motor functions in the B31 and B32 neurons of the buccal ganglia of Aplysia californica. J Neurophysiol 71:1514-1527.

Hurwitz I, Neustadter D, Morton DW, Chiel HJ, Susswein AJ (1996) Activity patterns of the B31/B32 pattern initiators innervating the I2 muscle of the buccal mass during normal feeding movements in Aplysia californica. J Neurophysiol 75:1309-1326.

Jing J, Weiss KR (2001) Neural mechanisms of motor program switching in Aplysia. J Neurosci 21:7349-7362.

Jing J, Weiss KR (2002) Interneuronal basis of the generation of related but distinct motor programs in Aplysia: implications for current neuronal models of vertebrate intralimb coordination. J Neurosci 22:6228-6238.

Jing J, Cropper EC, Hurwitz I, Weiss KR (2004) The construction of movement with behavior-specific and behavior-independent modules. J Neurosci 24:6315-6325.

Jordan R, Cohen KP, Kirk MD (1993) Control of intrinsic buccal muscles by motoneurons B11, B15, and B16 in Aplysia californica. J Exp Zool 265:496-506.

Kabotyanski EA, Baxter DA, Byrne JH (1998) Identification and characterization of catecholaminergic neuron B65, which initiates and modifies patterned activity in the buccal ganglia of Aplysia. J Neurophysiol 79:605-621.

Kabotyanski EA, Baxter DA, Cushman SJ, Byrne JH (2000) Modulation of fictive feeding by dopamine and serotonin in Aplysia. J Neurophysiol 83:374-392.

Kupfermann I (1974) Feeding behavior in Aplysia: a simple system for the study of motivation. Behav Biol 10:1-26.

Kuslansky B, Weiss KR, Kupfermann I (1978) A neural pathway mediating satiation of feeding behavior in Aplysia. Behav Biol 23:230-237.

Morgan PT, Jing J, Vilim FS, Weiss KR (2002) Interneuronal and peptidergic control of motor pattern switching in Aplysia. J Neurophysiol 87:49-61.

Morton DW, Chiel HJ (1993a) In vivo buccal nerve activity that distinguishes ingestion from rejection can be used to predict behavioral transitions in Aplysia. J Comp Physiol [A] 172:17-32.

Morton DW, Chiel HJ (1993b) The timing of activity in motor neurons that produce radula movements distinguishes ingestion from rejection in Aplysia. J Comp Physiol [A] 173:519-536.

Nargeot R, Baxter DA, Byrne JH (1997) Contingent-dependent enhancement of rhythmic motor patterns: an in vitro analog of operant conditioning. J Neurosci 17:8093-8105.

Neustadter DM, Drushel RF, Crago PE, Adams BW, Chiel HJ (2002) A kinematic model of swallowing in Aplysia californica based on radula/odon- 
tophore kinematics and in vivo magnetic resonance images. J Exp Biol 205:3177-3206

Orekhova IV, Jing J, Brezina V, DiCaprio RA, Weiss KR, Cropper EC (2001) Sonometric measurements of motor-neuron-evoked movements of an internal feeding structure (the radula) in Aplysia. J Neurophysiol 86:1057-1061.

Proekt A, Weiss KR (2003) Convergent mechanisms mediate preparatory states and repetition priming in the feeding network of Aplysia. J Neurosci 23:4029-4033.

Proekt A, Brezina V, Weiss KR (2004) Dynamical basis of intentions and expectations in a simple neuronal network. Proc Natl Acad Sci USA 101:9447-9452.

Scott ML, Govind CK, Kirk MD (1991) Neuromuscular organization of the buccal system in Aplysia californica. J Comp Neurol 312:207-222.

Susswein AJ, Byrne JH (1988) Identification and characterization of neurons initiating patterned neural activity in the buccal ganglia of Aplysia. J Neurosci 8:2049-2061.

Susswein AJ, Weiss KR, Kupfermann I (1978) The effects of food arousal on the latency of biting in Aplysia. J Comp Physiol 123:31-41.
Susswein AJ, Rosen SC, Gapon S, Kupfermann I (1996) Characterization of buccal motor programs elicited by a cholinergic agonist applied to the cerebral ganglion of Aplysia californica. J Comp Physiol [A] 179:509-524.

Warman EN, Chiel HJ (1995) A new technique for chronic single-unit extracellular recording in freely behaving animals using pipette electrodes. J Neurosci Methods 57:161-169.

Weiss KR, Mandelbaum DE, Schonberg M, Kupfermann I (1979) Modulation of buccal muscle contractility by serotonergic metacerebral cells in Aplysia: evidence for a role of cyclic adenosine monophosphate. J Neurophysiol 42:791-803.

Weiss KR, Chiel HJ, Koch U, Kupfermann I (1986) Activity of an identified histaminergic neuron, and its possible role in arousal of feeding behavior in semi-intact Aplysia. J Neurosci 6:2403-2415.

Weiss KR, Brezina V, Cropper EC, Hooper SL, Miller MW, Probst WC, Vilim FS, Kupfermann I (1992) Peptidergic cotransmission in Aplysia: functional implications for rhythmic behaviors. Experientia 48:456-463. 